\title{
Actualización en malformaciones venosas
}

\section{Current management of venous malformations}

\author{
P. Redondo, L. Aguado
}

\section{RESUMEN}

Las malformaciones venosas representan $2 / 3$ de todas las malformaciones vasculares y con frecuencia son mucho más complejas de lo que realmente parecen. Los pacientes con grandes malformaciones venosas requieren un estudio analítico y radiológico profundo, así como tratamiento específico para controlar una posible coagulación intravascular localizada. Si las lesiones son extensas, especialmente en el miembro inferior, se debe estudiar la presencia de una osteoporosis subyacente con la idea de prevenir fracturas patológicas. Igualmente debe controlarse la artropatía, y valorar la realización de una sinovectomía profiláctica precoz cuando la extensión radiológica lo aconseje, con la idea de evitar daños articulares irreversibles con el paso de los años. Actualmente la escleroterapia con microespuma se preconiza como el tratamiento de elección para las malformaciones vasculares de bajo flujo. En un futuro no muy lejano la utilización de fármacos antiangiogénicos selectivos, además de las heparinas de bajo peso molecular, servirán para controlar y tratar las malformaciones venosas.

Palabras clave. Malformación venosa. Coagulación. Angiogénesis. Escleroterapia. Microespuma.

\begin{abstract}
Venous malformations represent $2 / 3$ rds of all vascular malformations and are frequently much more complex than they appear to be. Patients with large venous malformations require a deep analytical and radiological study, as well as specific treatment to control any possible localised intravascular coagulation. If the lesions are extensive, especially in the lower member, a study should be made to detect the presence of an underlying osteoporosis with the idea of preventing pathological fractures. Equally, a check must be made for arthropathy, and an early prophylactic synovectomy must be considered when the radiological extension makes this advisable, with the idea of avoiding irreversible damage to the joints with the passage of time. Currently, microfoam scleropathy is favoured as the treatment of choice for low-flow vascular malformations. In the not too distant future, the use of selective antiangiogenic medicines, besides low-molecularweight heparins, will serve to control and treat venous malformations.
\end{abstract}

Key words. Venous malformation. Coagulation. Angiogenesis. Scleropathy. Microfoam.
Departamento de Dermatología.

Clínica Universidad de Navarra.

Pamplona.

Recepción: 8 de octubre de 2010

Aceptación provisional: 11 de noviembre de 2010

Aceptación definitiva: 24 de noviembre de 2010

\author{
Correspondencia \\ Dr. Pedro Redondo \\ Departamento de Dermatología \\ Clínica Universidad de Navarra \\ Avda. Pío XII, s/n \\ 31008 Pamplona \\ E-mail: predondo@unav.es
}




\section{INTRODUCCIÓN}

Las malformaciones vasculares son lesiones benignas no tumorales presentes siempre desde el nacimiento aunque a veces no son visibles hasta semanas o meses después. Su incidencia es de 1,5\%; aproximadamente $2 / 3$ partes son de predo- minio venoso y no distinguen entre sexo y raza $^{1}$. En el año 1982 Mulliken y Glowacki ${ }^{2}$ publicaron la clasificación biológica de las lesiones vasculares basada en las características del endotelio predominante; esta clasificación, ya clásica, se revisa cada 2 años por la International Society for the Study of Vascular Anomalies (ISSVA) (Tabla 1).

Tabla 1. Classificacion modificada de la International Society for the Study of Vascular Anomalies (Roma, Italia, 1996)

\begin{tabular}{|c|c|}
\hline & Tumores \\
\hline \multirow[t]{3}{*}{ Hemangiomas } & - Superficial (hemangioma capilar o hemangioma en fresa) \\
\hline & - Profundo (hemangioma cavernoso) \\
\hline & - Combinado \\
\hline \multirow[t]{7}{*}{ Otros } & - Hemangioendotelioma kaposiforme \\
\hline & - Angioma en penacho \\
\hline & - Hemangiopericitoma \\
\hline & • Hemangioendotelioma \\
\hline & - Granuloma piogénico \\
\hline & - Sarcoma de Kaposi \\
\hline & - Angiosarcoma \\
\hline \multicolumn{2}{|r|}{ Malformaciones vasculares } \\
\hline \multicolumn{2}{|c|}{ Capilares (C) (mancha en vino de Oporto) } \\
\hline \multicolumn{2}{|c|}{ Linfaticas (L) (linfangioma, higroma quístico) } \\
\hline \multicolumn{2}{|l|}{ Arteriovenosas (AV) } \\
\hline \multirow[t]{7}{*}{ Venosas (V) - Puras } & - Simples o localizadas \\
\hline & - Extensas o difusas \\
\hline & - CLV (incluye casi todos los S. de Klippel-Trenaunay) \\
\hline & - CV (incluye algunos S. de Klippel-Trenaunay) \\
\hline & $\bullet \mathrm{LV}$ \\
\hline & - CAV \\
\hline & - CLAV \\
\hline
\end{tabular}


Dentro de las malformaciones vasculares, las venosas han seguido múltiples clasificaciones. Una de ellas las divide en 4 grupos: a) malformaciones aisladas sin drenaje periférico, b) malformaciones que drenan en venas normales, c) malformaciones que drenan en venas displásicas y d) ectasias venosas. Aunque otros autores las diferencian en superficiales o profundas, localizadas, multicéntricas o difusas, nosotros preferimos la habitual separación de malformaciones venosas en puras y combinadas, siendo el principal exponente de este último grupo el síndrome de KlippelTrenaunay. Las malformaciones venosas puras a su vez pueden dividirse en simples o localizadas y extensas o difusas.

\section{ETIOPATOGENIA}

Las malformaciones venosas se deben a errores difusos o localizados del desarrollo embriológico, clásicamente atribuidos a mutaciones esporádicas. Sin embargo, una serie de publicaciones recientes habla a favor de un posible carácter familiar hereditario. Vikkula y cols. han encontrado una mutación caracterizada por un aumento de la actividad del receptor de la tirosincinasa-Tie-2 en dos familias con malformaciones venosas ${ }^{3}$. Tie-2 juega un papel esencial para el desarrollo temprano de los vasos y un incremento de su actividad puede producir un crecimiento anómalo del plexo vascular primario. Este mismo grupo de trabajo también ha definido la implicación de la glomulina en pacientes con malformaciones glomuvenosas ${ }^{4}$. Asimismo se conocen algunas mutaciones genéticas en malformaciones cavernosas cerebrales ${ }^{5}$ y en malformaciones vasculares combinadas tipo síndrome de Klippel-Trenaunay ${ }^{6}$.

Las malformaciones venosas son lesiones de bajo flujo, hemodinámicamente inactivas, que están presentes desde el nacimiento y progresivamente empeoran a lo largo de la vida del paciente. Aunque sea muy lentamente las lesiones van creciendo y expandiéndose de forma insidiosa. Si bien la patogénesis no está bien clarificada, su formación y progresión están estrechamente relacionadas con la angiogénesis?
La angiogénesis constituye un proceso complejo, regulado por muchos factores proangiogénicos y antiangiogénicos que conlleva la formación de una nueva vasculatura funcional y que incluye la diferenciación de células endoteliales y murales (pericitos), la proliferación y migración celular y la especificación de vasos arteriales, venosos o linfáticos.

La angiopoietina-1 (Ang-1) y la Ang2 son los reguladores más potentes de la neovascularización. En presencia del factor de crecimiento del endotelio vascular (VEGF), la Ang-2 promueve un incremento rápido del diámetro capilar, una remodelación de la membrana basal y proliferación y migración de células endoteliales que estimulan la formación de nuevos vasos ${ }^{8}$. La Ang-2 se localiza en los cuerpos endoteliales de Webel-Palade y es rápidamente liberada tras la activación endotelial. La Ang es ligando de la tirosincinasa Tie-2 que actúa como un regulador crítico en la estabilización y maduración de nuevos vasos ${ }^{9}$. Recientemente hemos apreciado en una serie de 31 pacientes (11 malformaciones venosas extensas y 20 síndromes de Klippel-Trenaunay) un incremento sérico de los niveles de Tie-2, metaloproteinasa-9 y Ang-2, así como de los niveles plasmáticos de dímero-D, activador tisular del plasminógeno, inhibidor del activador del plasminógeno y factor Von-Willebrand respecto a controles sanos ${ }^{10}$. En los pacientes había una disminución sérica significativa de la Ang-1 y factor de crecimiento derivado de plaquetas $\mathrm{AB}$ y $\mathrm{BB}$ respecto a los controles. Estos hallazgos parecen sugerir una alteración del sistema Ang-Tie-2 que puede producir vasos anómalos dilatados con insuficiente componente de células murales ${ }^{10}$.

La interacción entre la angiogénesis y la coagulación ha sido descrita en varios estudios. La angiogénesis y la trombosis están estrechamente unidas; por ejemplo las plaquetas contienen y liberan VEGF, lo que favorece la hipótesis que implica a las plaquetas localizadas en los microtrombos locales en la formación de nuevos vasos ${ }^{11}$. Otras líneas de investigación destacan el papel esencial de la trombina para favorecer la angiogénesis. Datos in vitro demuestran 
como la trombina estimula varias vías angiogénicas, incluyendo metaloproteinasas de matriz, VEGF, receptores de VEGF, Ang2 e integrina $\alpha v-\beta 3$. La plasmina interviene directamente en la ruptura de la membrana basal y en el proceso angiogénico de degradación de la matriz extracelular, con el sistema del activador del plasminógeno jugando un papel esencial en la síntesis de plasmina. De esta manera el desarrollo y lenta proliferación de las malformaciones venosas puede ser influido por el sistema fibrinolítico que interactúa con la angiogénesis.

Las heparinas de bajo peso molecular tienen un efecto antiangiogénico al inhibir la unión de factores de crecimiento angiogénicos sobre sus receptores. La activación de angiogénesis y coagulación en pacientes con cáncer puede explicar algunos casos de progresión tumoral y desarrollo de metástasis. Así, algunos autores sugieren que la terapia con heparina de bajo peso molecular puede prolongar la supervivencia en pacientes oncológicos con y sin tromboembolismos venosos ${ }^{12}$. Los niveles plasmáticos de dímero-D pueden ser útiles para monitorizar la terapia anticancerosa de la heparina en estos pacientes. Extrapolando los datos anteriores se puede hipotetizar que la heparina de bajo peso molecular, además de su acción anticoagulante puede tener un efecto antiangiogénico sobre las malformaciones venosas controlando el desarrollo y expansión de nuevos vasos ${ }^{9}$.

\section{CLÍNICA}

\section{Malformaciones venosas puras}

Las malformaciones venosas están constituidas por vasos ectásicos de bajo flujo sanguíneo, morfológica e histológicamente similares a las venas. La piel o mucosa que las recubre varía de color en función de la profundidad y el grado de ectasia de la lesión. Las más superficiales son de color morado y las profundas más azuladas, verdosas o incluso imperceptibles. Se trata de lesiones blandas al tacto, a veces de aspecto nodular, que se vacían mediante la compresión (Fig. 1). En determinadas posiciones la malformación se rellena de sangre, como por ejemplo las localizadas en cabeza y cuello tras realizar una maniobra de Valsalva, y en otras se vacía cuando se eleva la zona afecta por encima del nivel del corazón. En las lesiones muy ectásicas no es rara la presencia de pequeñas trombosis venosas, que pueden inflamarse y producir dolor e inflamación, y de flebolitos que son marcadores radiológicos de este tipo de malformaciones y aparecen a edades tempranas. También son especialmente característicos el dolor matutino que va disminuyendo con el movimiento y la exacerbación sintomática en las mujeres con el embarazo y los cambios hormona$\operatorname{les}^{1,13}$.
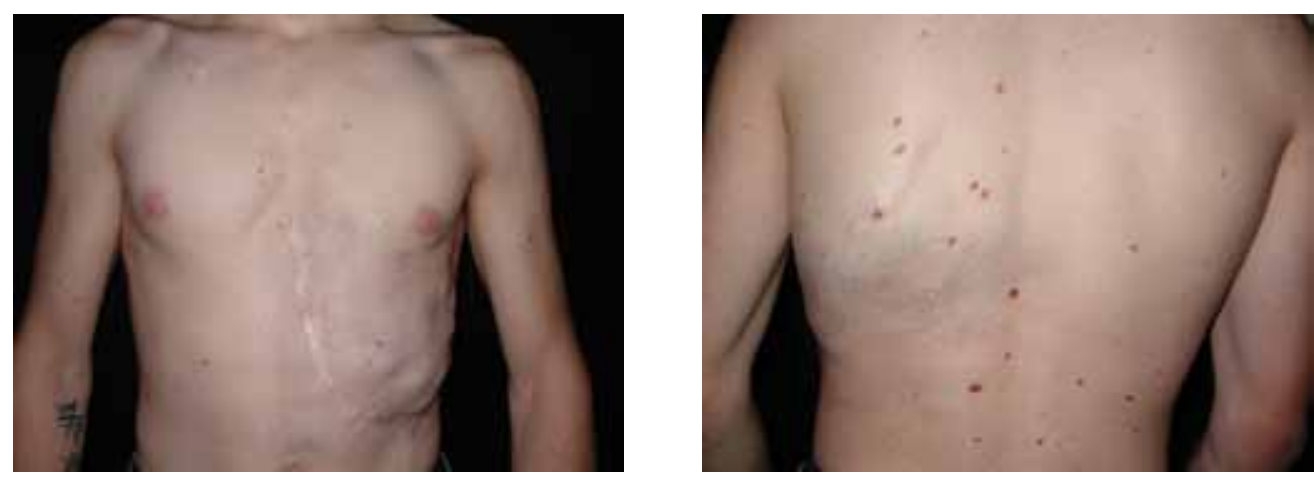

Figura 1. Paciente varón con malformación venosa extensa en región toraco-abdominal izquierda, con un patrón metamérico que se extiende hacia la espalda. Se aprecia cicatriz secundaria a cirugía en la infancia. 
En el polo cefálico las malformaciones venosas con frecuencia presentan compromiso mucoso, afectan la lengua, el paladar, los labios y las mucosas yugales e infiltran músculo, glándulas salivares e incluso estructuras óseas. Cuando se extienden por la región periorbitaria o el cuello su crecimiento puede ocasionar compromiso ocular y obstrucción de la vía aérea respectivamente. Con relativa frecuencia las malformaciones venosas asientan en los miembros, muchas veces con una extensión lineal y profunda mayor que la apariencia externa. Prácticamente siempre hay afectación muscular y no es raro el compromiso articular y óseo.

\section{Cuadros con entidad propia}

Dentro de las malformaciones venosas puras existen diversos subgrupos, destacando las malformaciones venosas cutáneo-mucosas familiares, las malformaciones glomuvenosas, las malformaciones venosas cerebrales y el síndrome del nevus azul en tetina de goma.

Las malformaciones venosas cutáneomucosas familiares son debidas a una mutación en el receptor Tie-2 que se hereda con un patrón autosómico dominante. ${ }^{3}$ Clínicamente se caracterizan por la presencia de múltiples lesiones venosas de pequeño tamaño que afectan a la piel y a las mucosas.

Las malformaciones glomuvenosas representan el 5\% de todas las malformaciones venosas. El $63 \%$ de los casos se trata de lesiones hereditarias donde se ha descrito la mutación de la glomulina (locus p-21 del cromosona 9) ${ }^{4}$. Histológicamente las lesiones se caracterizan por vasos interconectados por cordones de células glómicas. Las lesiones pueden ser localizadas, diseminadas o metaméricas y se manifiestan como lesiones nodulares o en forma de placas, rosadas o azul oscuras, con una apariencia en empedrado y a veces áreas hiperqueratósicas. Clínicamente se diferencian de las malformaciones venosas porque las glomuvenosas se encuentran limitadas a la piel y al tejido celular subcutáneo, nunca son infiltrantes, no son compresibles, no se vacían levantándolas por encima del corazón, no tienen flebolitos y no asocian alteraciones de la coagulación ${ }^{14}$. De hecho, hoy en día la tendencia es a clasificarlas como un grupo diferente a las malformaciones venosas.

Las malformaciones venosas cerebrales, también denominadas angiomas cavernosos, presentan un carácter familiar con el mismo patrón hereditario que las anteriores. Un subgrupo de estos pacientes tiene lesiones cutáneas capilares venosas de aspecto hiperqueratósico y con frecuencia pueden asociar una malformación venosa ocular. En algunas familias afectas se ha detectado una anomalía en 7q11-22 ${ }^{15}$.

El síndrome del nevus azul en tetina de goma es una entidad que asocia múltiples malformaciones venosas en la piel y el tracto gastrointestinal. Las lesiones cutáneas, que generalmente están presentes en el momento del nacimiento o bien aparecen de forma progresiva en la primera infancia, son nódulos de pequeño tamaño, azulados o purpúricos. En ocasiones se trata de tumoraciones más extensas o máculas azuladas, característicamente depresibles a la palpación en forma de tetina de goma ${ }^{16}$. En el tubo digestivo la localización principal es el intestino delgado. Estas malformaciones pueden causar hemorragia digestiva con anemia secundaria que requiere control endoscópico y tratamiento quirúrgico en función de su gravedad. Desde el punto de vista histopatológico se trata de ectasias vasculares de forma y tamaño irregular localizadas en la dermis profunda y el tejido celular subcutáneo. La mayoría de los casos publicados son esporádicos aunque en algunos se ha descrito un patrón de herencia autosómico dominante.

\section{Malformaciones combinadas}

El síndrome de Klippel-Trenaunay se caracteriza por la asociación de una malformación vascular capilar, linfática y venosa junto con hipertrofia esquelética y aumento de tejidos blandos de uno o más miembros. Aunque la presentación suele ser de 
aparición esporádica, recientemente se han descrito casos familiares con mutaciones genéticas ${ }^{6}$. La malformación capilar o mancha en vino de Oporto es única o múltiple, afecta generalmente a una extremidad inferior (95\% de los casos) y en más de un $10 \%$ de los pacientes la lesión sobrepasa el miembro y aparece afectación del tronco e incluso hemicorporal ${ }^{17}$. Una publicación reciente distingue entre manchas de bordes geográficos (contorno irregular bien definido, asemejando a un continente) de coloración rojiza intensa o violácea, de otras más difusas con una distribución segmentaria de coloración rosada. Las primeras presentan más afectación linfática y conllevan un mayor número de complicaciones ${ }^{18}$. En una serie pequeña pero significativa hemos apreciado cómo las manchas geográficas también se asocian con más frecuencia a hipoplasia o aplasia del sistema venoso profundo $^{19}$. Otro trabajo de nuestro grupo, tras estudiar 51 pacientes consecutivos con síndrome de Klippel-Trenaunay ha demostrado en 9 pacientes cómo la presencia de malformaciones en manos y pies (macro o microdactilias, ectrodactilia, sindactilia, clinodactilia y cantodactilia) va unida de forma significativa a alteraciones del sistema venoso profundo (aplasia, hipoplasia o duplicación $)^{20}$.

La malformación venosa del síndrome de Klippel-Trenaunay se manifiesta como venas laterales anómalas o venas embrionarias persistentes, que son prominentes por incompetencia valvular y por la frecuente alteración asociada al sistema venoso profundo. Aunque se pueden observar desde el nacimiento, aparecen con más frecuencia cuando el paciente inicia la deambulación.

El incremento de volumen y tamaño en la extremidad afecta va haciéndose llamativo con la edad destacando un aumento de tejidos blandos que requerirá seguimiento y tratamiento específicos, aunque en un pequeño porcentaje de pacientes hay atrofia y acortamiento de la extremidad. La dismetría del miembro fundamentalmente con hipertrofia ha sido reportada en el $66 \%$ de los pacientes con síndrome de Klippel-Trenaunay, siendo la extensión de la malformación vascular el único factor asociado de riesgo independiente. En recién nacidos, o pacientes que no alcancen el año de edad con síndrome de Klippel-Trenaunay, aunque no presenten dismetría del miembro, la realización de un Eco-doppler y el hallazgo de una diferencia en el flujo arterial entre el miembro afecto y el contralateral superior al $50 \%$ puede predecir una futura dismetría, y por tanto obligar a un seguimiento más estrecho que en pacientes con un flujo similar en ambas extremidades inferiores ${ }^{21}$.

\section{Complicaciones con repercusión sistémica}

En una serie de 27 pacientes con malformaciones venosas extensas de miembros, todos presentaban afectación muscular, el $81 \%$ compromiso articular (del codo o la rodilla) y el $63 \%$ de 19 pacientes estudiados tenían alteraciones óseas (adelgazamiento, desmineralización o cambios líticos) ${ }^{22}$. Otro grupo ha demostrado en el 71\% de los pacientes la desmineralización ósea local que puede asociarse a fracturas patológi$\mathrm{cas}^{23}$. Al contrario que las malformaciones vasculares combinadas tipo síndrome de Klippel-Trenaunay, en las malformaciones venosas no suele haber hipertrofia músculo-esquelética de la extremidad afecta sino más bien atrofia o hipotrofia (Fig. 2). Cuando está englobada la rodilla es frecuente la gonalgia con limitación funcional y hematrosis, y no es raro que la artropatía por hemosiderina acabe evolucionando a una artritis degenerativa, progresivamente irreversible, similar a la que aparece en los síndromes hemofílicos ${ }^{24}$.

Las grandes malformaciones venosas de miembros obligan a descartar una extensión al tronco y una afectación visceral, concretamente las de miembros superiores a pleura, mediastino y pulmón y las de miembros inferiores a pelvis y cavidad abdominal. El sangrado gastrointestinal o genitourinario es el síntoma más frecuente en los pacientes que tienen extensión visceral de la malformación venosa ${ }^{25}$. 


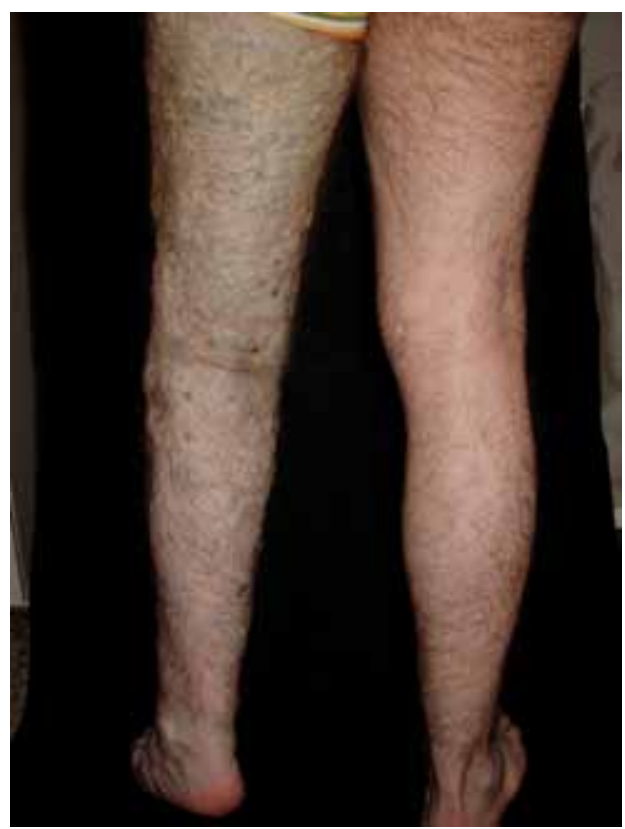

En las malformaciones venosas puras o combinadas se han descrito trastornos de la coagulación. Concretamente una coagulación intravascular localizada, caracterizada por un consumo local de factores de la coagulación dentro de la malformación o secundario al éstasis venoso, como consecuencia del cual se forman los microtrombos y los flebolitos. Analíticamente el fibrinógeno suele estar bajo (menor de $0,1 \mathrm{~g} / \mathrm{l}$ ) y el dímero-D elevado ${ }^{26,27}$. En un estudio reciente de 280 pacientes con malformaciones vasculares, 195 de ellos $(69,6 \%)$ tenían malformaciones venosas, mientras que 85 pacientes presentaban otro tipo de lesiones ${ }^{28}$. En el primer grupo, en 83 pacientes la detección plasmática de dímero-D fue muy alta, mientras que en el grupo de las no venosas, sólo en 3 pacientes el dímero-D estaba elevado. La sensibilidad fue 42,6\% (95CI, 35,6-49,5\%) y la especificidad $96,5 \%$ (95 CI-92,5-100\%). El estudio concluye que el dímero-D es muy específico de malformaciones venosas, ya sean puras, combinadas o sindrómicas y que este test analítico puede ser muy útil para confirmar el diagnóstico clínico, así como para el diagnóstico diferencial res-

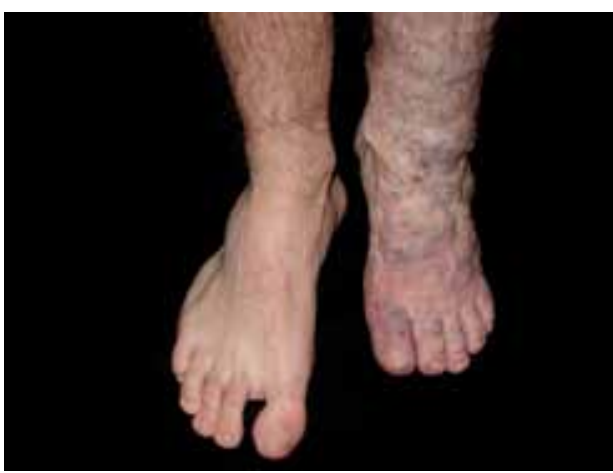

Figura 2. Malformación venosa extensa que engloba todo el perímetro de la extremidad inferior. Nótese el acortamiento respecto a la extremidad contralateral y la disminución de tamaño del pie afecto.

pecto a malformaciones glomuvenosas $\mathrm{y}$ malformaciones linfáticas. También puede ayudar a diferenciar malformaciones combinadas entre sí, en concreto un síndrome de Klippel-Trenaunay respecto a un síndrome de Parkes-Weber. La determinación basal del dímero-D, es especialmente útil antes de realizar un procedimiento terapéutico. Así, en los pacientes en los que está elevado, la retirada de las medias de compresión, la realización de cirugía o escleroterapia pueden empeorar el cuadro predisponiendo a un posible tromboembolismo, por lo que debe iniciarse anticoagulación con heparina de bajo peso molecular. Con esa profilaxis también se reduce el elevado riesgo de sangrado durante un procedimiento quirúrgico. La coagulación intravascular localizada es diferente de la coagulación intravascular diseminada o síndrome de Kasabach-Merrit, caracterizado por anemia hemolítica, trombopenia y coagulapatía. Este último cuadro es específico de algunos tumores vasculares como el hemangioendotelioma kaposiforme o el hemangioma en penacho pero no de los hemangiomas comunes ni de ningún tipo de malformación vascular ${ }^{29}$. 
Por otra parte, en pacientes con malformaciones venosas extensas en extremidades con un patrón segmentario y en las malformaciones combinadas tipo síndrome de Klippel-Trenaunay, pueden existir anomalías del sistema venoso profundo en casi el 50\% de los casos. Estos hallazgos obligan a realizar un estudio del sistema venoso profundo del miembro afecto, sobre todo antes de realizar un procedimiento terapéutico, por los posibles efectos secundarios que puedan derivarse $\mathrm{e}^{30}$.

La principal complicación del síndrome de Klippel-Trenaunay es la tromboflebitis que ocurre en el $20-45 \%$ de los casos y ocasiona embolismos pulmonares en el $4-25 \%$ de los pacientes ${ }^{31}$. De hecho existen publicaciones anecdóticas de pacientes con síndrome de Klippel-Trenaunay con hipertensión pulmonar atribuible a estos embolismos $^{32}$. En condiciones normales se considera que una presión pulmonar arterial sistólica menor de 36 es normal, mientras que los valores entre 36 y $50 \mathrm{mmHg}$ son borderline, y valores superiores a 50 $\mathrm{mmHg}$ claramente patológicos. Recientemente hemos estudiado 32 pacientes con malformaciones vasculares extensas (14 malformaciones venosas extensas y 18 síndromes de Klippel-Trenaunay) midiendo su presión pulmonar arterial sistólica y comparándola con 32 controles sanos ${ }^{33}$. En el grupo de pacientes la media de la presión pulmonar arterial sistólica fue de $42,16 \mathrm{mmHg}$ respecto a $27,69 \mathrm{mmHg}$ en el grupo control $(\mathrm{p}<0.001)$. También hemos demostrado una correlación positiva ( $\mathrm{r}$ : $0,522, \mathrm{p}<0,001)$ entre los niveles de dímeroD y la presión pulmonar arterial sistólica. Aunque el origen de esta patología parece ser atribuible a embolismos pulmonares, no hay que descartar la posibilidad de angiogénesis local e interacción de otros factores que ocasionen un aumento de la presión arterial pulmonar.

\section{DIAGNÓSTICO}

El diagnóstico de las malformaciones venosas cutáneas se basa en la historia clínica y la exploración física. Los estudios mediante técnicas de imagen encuentran su indicación en aquellos casos en los que existen dudas sobre la naturaleza de la lesión, siendo una herramienta complementaria para esclarecer y confirmar el diagnóstico.

La ecografía asociada al Doppler proporciona, además de información anatómica, datos hemodinámicos como la velocidad y dirección del flujo de gran utilidad en las malformaciones venosas.

La Resonancia Magnética es la técnica de elección para la valoración inicial de las malformaciones venosas, ya que permite delimitar la extensión completa de la lesión en diversos planos anatómicos de manera no invasiva. Las secuencias en T1 presentan una señal menor a la de la grasa adyacente, mientras que en $\mathrm{T} 2$ sucede lo contrario $^{34}$.

Especialmente en malformaciones localizadas en las extremidades, la flebografía mediante punción directa de los vasos anómalos, junto con la utilización de torniquetes para redirigir el flujo, va a permitir delimitar anatómicamente la extensión y componentes de la lesión, así como el volumen de los diversos compartimentos venosos. Sin duda, la flebografía sigue siendo la técnica con mayor rendimiento para estudiar la existencia y permeabilidad del sistema venoso profundo en miembros inferiores de pacientes con malformaciones venosas extensas o combinadas tipo síndrome de Klippel-Trenaunay. Hoy en día con los avances en el diagnóstico por imagen se considera que un angio-TAC o una angio-Resonancia en 3D puede ser la prueba de elección para estudiar a fondo una malformación vascular extensa localizada en una extremidad ${ }^{30}$. El angio-TAC indirecto tiene una mejor resolución espacial y detecta mejor las alteraciones óseas, frente a la angio-resonancia que es de elección en niños y embarazadas y en general en el seguimiento de los pacientes, al carecer de radiación ionizante ${ }^{35}$. Estas técnicas permiten conocer la localización exacta de la lesión en un plano tridimensional, detectando una posible infiltración músculo-esquelética y extensión torácica y/o 
abdominopélvica. Además permiten valorar cambios en la densidad ósea (osteoporosis) y dismetría, así como comprobar la existencia del sistema venoso profundo y su permeabilidad. También son de utilidad para detectar anormalidades del sistema venoso superficial, demostrar la presencia de venas aberrantes o ciáticas y determinar su extensión y vía de drenaje (Tabla 2). Aunque estos procedimientos siguen sien- do inferiores a la flebografía convencional al no proporcionar el mismo grado de información sobre la hemodinámica de la malformación, representan un avance muy significativo y en nuestra experiencia sólo será necesaria la complementación con procedimientos angiográficos invasivos en caso de hipoplasias vasculares donde no quede claro el funcionamiento hemodinámico del vaso.

Tabla 2. Diagnóstico y seguimiento de las malformaciones venosas puras o combinadas extensas.

\section{DIAGNÓSTICO}

- Eco-doppler + angio-RM indirecta en 3D o angio-TAC indirecto en 3D

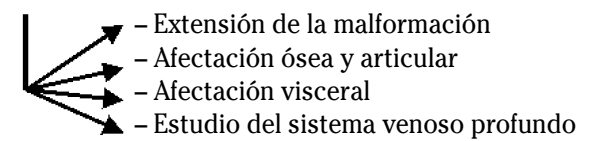

- Ecocardiograma

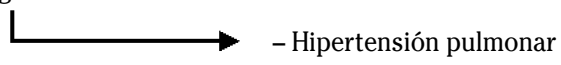

- Analítica (Dímero-D y fibrinógeno)

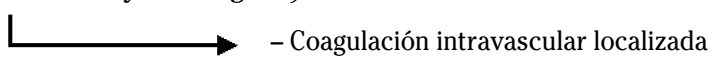

\section{SEGUIMIENTO}

- Con discrepancias en la longitud del miembro

$\longleftrightarrow$ - Evaluación cada 4-6 meses por cirugía ortopédica hasta la pubertad

- Con osteoporosis o afectación articular

$\longrightarrow$ - Radiografía simple, RM de rodilla o angio-RM/angio-TAC en 3D

- Con hipertensión pulmonar

$\longleftrightarrow$ - Ecocardiograma periódico. Cateterismo en función del grado

- Con coagulación intravascular localizada

$\longrightarrow$ - Analítica (dímero-D y fibrinógeno) cada 4-6 meses

\section{TRATAMIENTO}

Primum non nocere, lo primero no hacer daño, es la regla de oro en la práctica médica durante siglos, y sin duda un principio esencial para el manejo de las malformaciones venosas complejas.

Con frecuencia la extirpación quirúrgica puede ser el tratamiento estándar en malformaciones pequeñas, aunque no tiene eficacia en las malformaciones extensas donde, además de sangrado masivo, puede producir daños nerviosos motores irreparables.

La compresión con prendas adecuadas o vendajes específicos es, sin lugar a dudas, el tratamiento esencial de primera línea para el manejo diario de las malformaciones venosas, especialmente las que afectan a las extremidades. Regular la activación de la coagulación dentro de una malformación venosa tiene dos objetivos: por un lado controlar el dolor local y por otro prevenir las complicaciones hemorrágicas. La compre- 
sión elástica al limitar o reducir el estasis venoso disminuye la activación local de la coagulación. La administración médica de heparinas de bajo peso molecular reduce el consumo de factores de coagulación y por tanto el estado de coagulación intravascular localizada. Aunque por el riesgo de trombosis o sangrado no hay necesidad de tratar una coagulopatía intravascular localizada crónica si el fibrinógeno es normal y los niveles de dímero-D son elevados, sí debe tenerse en cuenta que el dolor intralesional de la malformación que ocurre en el $20 \%$ de los pacientes es debido a estas alteraciones ${ }^{9}$. En un trabajo reciente Dompmartin y cols. reportan resultados satisfactorios disminuyendo el dolor en 22 pacientes con malformaciones venosas extensas y niveles elevados de dímero-D a los que se les administró heparina de bajo peso molecular (enoxaparina 100 anti-Xa U/kd/día durante 20 días) ${ }^{28}$. El fármaco también reduce la hemorragia espontánea y las complicaciones hemorrágicas derivadas de una intervención quirúrgica, aunque a priori parezca contradictorio que un anticoagulante pueda ser útil para prevenir una tendencia hemorrágica. Los autores sugieren profilaxis preventiva con heparina de bajo peso molecular durante 10 días previo a cualquier procedimiento quirúrgico en estos pacientes.

De forma similar, cuando hay afectación músculo-esquelética u osteo-articular hay que prevenir el daño futuro a largo plazo potenciando la musculatura, intentando reducir la osteoporosis y progresiva desmineralización ósea con la administración de bifosfonatos o andrógenos ${ }^{9}$, así como prevenir una posible artropatía crónica mediante la realización de sinovectomía en una fase precoz ${ }^{36}$.

La embolización con coils o partículas no es el tratamiento de elección de las malformaciones venosas, pero sí puede ser eficaz en algunos pacientes para tratar vasos de gran calibre. En la misma línea, la radiofrecuencia con láser endovascular parece ser una terapia emergente en lesiones de morfología lineal.

Según nuestra experiencia y basados en los datos disponibles en la literatura, el tratamiento de elección de las malformaciones venosas es la escleroterapia ${ }^{37}$. La escleroterapia convencional con líquidos ofrece unos buenos resultados en malformaciones de pequeño tamaño, pero por sus intrínsecas limitaciones es inefectiva en las lesiones de mayor tamaño. En estos casos la escleroterapia con alcohol puede ser más eficaz, pero no está exenta de efectos secundarios importantes y requiere larga experiencia e infraestructura adecuada para su utilización. Hoy en día la introducción de esclerosantes en forma de microespuma ha transformado el escenario de la escleroterapia, y existen diversos artículos que demuestran la eficacia y seguridad de la escleroterapia con microespuma de polidocanol guiada por ultrasonidos en el manejo de malformaciones venosas puras y combinadas tipo síndrome de KlippelTrenaunay $^{38-41}$.

\section{BIBLIOGRAFÍA}

1. Redondo P. Clasificación de las anomalías vasculares (tumores y malformaciones). Características clínicas e historia natural. An Sist Sanit Navar 2004; 27 (Supl. 1): 9-25.

2. Mulliken JB, Glowacki J. Hemangiomas and vascular malformations in infants and children: a classification based on endothelial characteristics. Plast Reconstr Surg 1982; 69: 412-422.

3. Vikkula M, Boon L, Carraway KL 3Rd, Calvert JT, DiAmonti AJ, Goumnerov B et al. Vascular dysmorphogenesis caused by an activating mutation in the receptor tyrosine kinasa TIE2. Cell 1996; 87: 1181-1190.

4. Boon LM, Brouillard P, Irrthum A, Karttunen L, WARMAN ML, RUdolPH R et al. A gene for inherited cutaneous venous anomalies ("glomangiomas") localizes to chromosome 1p21-22. Am J Hum Genet 1999; 65: 125-133.

5. SAHoo T, Johnson EW, Thomas JW, Kuehl PM, Jones TL, DoKken CG et al. Mutations in the gene encoding KRIT1, a Krev-1/rapla binding protein, cause cerebral cavernous malformations (CCM1). Hum Mol Genet 1999; 8: 23252333.

6. Tian XL, Kadaba R, You SA, Liu M, Timur AA, YANG $\mathrm{L}$ et al. Identification of an angiogenic factor that when mutated causes susceptibility to Klippel-Trenaunay syndrome. Nature 2004; 427: 640-645. 
7. Marler J, Fishman SJ, Kilroy SM, Fang J, Upton J, MulLIKEn JB, et al. Increased expression of urinary matrix metalloproteinases parallels the extent and activity of vascular anomalies. Pediatrics 2005; 116: 38-45.

8. Lobov IB, Brooks PC, LANG RA. Angiopoietin-2 displays VEGF-dependent modulation of capillary structure and endothelial cell survival in vivo. Proc Natl Acad Sci U S A 2002; 99 : 11205-11210.

9. REDONDo P. The hidden face of venous malformations. Arch Dermatol 2008; 144: 922-926.

10. Redondo P, Aguado L, Marquina M, Paramo JA, Sierra A, Sánchez-Ibarrola A et al. Angiogenic and prothrombotic markers in extensive slow-flow malformations: implications for antiangiogenic/antithrombotic strategies. $\mathrm{Br}$ J Dermatol 2010; 162: 350-356.

11. Kisucka J, Butterfield CE, Duda DG et al. Platelets and platelet adhesion support angiogenesis while preventing excessive hemorrhage. Proc Natl Acad Sci U S A 2006; 103: 855-860.

12. Di Nisio M, Klerk CP, Meijers JC, Buller HR. The prognostic value of the D-dimer test in cancer patients treated with and without low-molecular-weight heparin. J Thromb Haemost 2005; 3: 1531-1533.

13. Redondo P. Malformaciones vasculares (I). Concepto, clasificación fisiopatogenia y manifestaciones clínicas. Actas Dermosifiliogr 2007; 98: 141-158.

14. Boon LM, Mulliken JB, Enjolras O, VikKula M. Glomuvenous malformation (Glomangioma) and venous malformation distinct clinicopathologic and genetic entities. Arch Dermatol 2004; 140: 971-976.

15. Gil-Nagel A, Dubovsky J, Wilcox KJ, Stewart JM, ANDERSON VE, LEPPIK IE et al. Familial cerebral cavernous angioma: a gene localized to a 15cM interval on chromosome 7q. Ann Neurol 1996; 39: 311-314.

16. Oranje AP. Blue rubber bleb nevus syndrome. Pediatr Dermatol 1986; 3: 304-310.

17. Jacob AG, Driscoll DJ, Shaughnessy WJ, Stanson AW, Clay RP, GLOVICZKI P et al. Klippel-Trenaunay syndrome: spectrum and management. Mayo Clin Proc 1998; 73: 28-36.

18. MAARI C, FRIEDEN IJ. Klippel-Trenaunay syndrome: the importance of "geographic stains" in identifying lymphatic disease and risk of complications. J Am Acad Dermatol 2004; 51: 391-398.

19. Bastarrika G, Redondo P, Sierra, Cano D, MartínezCuesta A, López-Gutierrez JC et al. New techni- ques for the evaluation and therapeutic planning of patients with Klippel-Trenaunay syndrome. J Am Acad Dermatol 2007; 56: 242-249.

20. Redondo P, Bastarrika G, Aguado L, MartínezCuesta A, Sierra A, Cabrera J et al. Foot or hand malformations related to deep venous system anomalies of the lower limb in KlippelTrenaunay syndrome. J Am Acad Dermatol 2009; 61: 621-628.

21. Samimi M, Maruani A, Bertrand P, Arbeille P LoRetTe G. Arterial blood flow in limbs with port-wine stains can predict length discrepancy. Br J Dermatol 2009; 160: 219-220.

22. Enjolras O, Ciabrini D, Mazoyer E, Laurian C, Herbreteau D. Extensive pure venous malformations in the upper or lower limb, a review of 27 cases. J Am Acad Dermatol 1997; 36: 219-225.

23. Boyd JB, Mulliken JB, Kaban LB, Upton J, MuRRAY JE. Skeletal changes associated with vascular malformations. Plast Reconstr Surg 1984; 76: 789-797.

24. Bonaga S, Bardi C, Gigante C, Turra S. Synovial involvement in hemangiomatosis. Arch Orthop Trauma Surg 2003; 123: 102-106.

25. Redondo P, Bastarrika G. Radiologic and serologic features of extensive venous malformations associated with atrophy, osteoporosis, and visceral involvement: implications for future management. Dermatol Surg 2008; 34: 1718-1725

26. Dompmartin A, Acher A, Thibon P, Tourbach S, Hermans C, Deneys V et al. Association of localized intravascular coagulopathy with venous malformations. Arch Dermatol 2008; 144: 873-877.

27. Mazoyer E, Enjolras O, Bisdorff A, Perdu J, WasSEF M, DrouET L. Coagulation disorders in patients with venous malformation of the limbs and trunk: a case series of 118 patients. Arch Dermatol 2008; 144: 861-867.

28. Dompmartin A, Ballieux F, Thibon P, Lequerrec A, Hermans C, Clapuyt P et al. Elevated D-dimer level in the differential diagnosis of venous malformations. Arch Dermatol 2009; 145: 1239-1244.

29. Enjolras O, Wassef M, Mazoyer E, Frieden IJ, RIEU PN, Drouet L et al. Infants with Kasabach-Merritt syndrome do not have "true" hemangiomas. J Pediatr 1997; 130: 631-640.

30. Eifert S, Villavicencio JL, Kao TC, Taute BM, RIcH NM. Prevalence of deep venous anomalies in congenital vascular malformations of venous predominance. J Vasc Surg 2000; 31: $462-471$. 
31. SAmuel M, SpITz L. Klippel-Trenaunay syndrome: clinical features, complications and management in children. Br J Surg 1995; 82: 757-761.

32. Muluk SC, Ginns LC, Semigran MJ, Kaufman JA, GERTLER JP. Klippel- Trenaunay syndrome with multiple pulmonary emboli: an unusual cause of progressive pulmonary dysfunction. J Vasc Surg 1995; 21: 686-690.

33. Rodriguez M, Aguado L, Redondo P. Pulmonary arterial hypertension in patients with slowflow vascular malformations (extensive venous malformations or Klippel-Trenaunay syndrome). Arch Dermatol 2010 (en prensa).

34. REDONDo P. Malformaciones vasculares (II). Diagnóstico, histopatología y tratamiento. Actas Dermosifiliogr 2007; 98: 219-35

35. Bastarrika G, Redondo P. Response: Multidetector row computed tomography arteriography in the preoperative assessment of patients with Klippel-Trenaunay syndrome. doi:10.1016/j.jaad. 2008. 10. 038
36. Johnson JN, Shaughnessy WJ, Stans AA, UnRuh KP, Sim FH, McInTosh AL et al. Management of knee arthropathy in patients with vascular malformations. J Pediatr Orthop 2009; 29: 380-384.

37. Cabrera J, Redondo P. Tratamiento esclerosante de las malformaciones vasculares. An Sist Sanit Navar 2004; 27 (Supl. 1): 117-126.

38. Cabrera J, Cabrera J Jr, Garcia-Olmedo MA, ReDONDO P. Treatment of venous malformations with sclerosant in microfoam form. Arch Dermatol 2003; 139: 1409-1416.

39. Hsu TS, WeIss RA. Foam sclerotherapy: a new era. Arch Dermatol 2003; 139: 1494-1496.

40. NitecKI S, BASS A. Ultrasound-guided foam sclerotherapy in patients with Klippel- Trenaunay syndrome. Isr Med Assoc J 2007; 9: 72-75.

41. Bergan J, Pascarella L, Mekenas L. Venous disorders: treatment with sclerosant foam. J Cardiovasc Surg (Torino) 2006; 47:9-18. 